\title{
Robustness of Entanglement for Bell Decomposable
}

\author{
States \\ S. J. Akhtarshenas ${ }^{a, b, c *}$, M. A. Jafarizadeh ${ }^{a, b, c \dagger}$ \\ ${ }^{a}$ Department of Theoretical Physics and Astrophysics, Tabriz University, Tabriz 51664, Iran. \\ ${ }^{b}$ Institute for Studies in Theoretical Physics and Mathematics, Tehran 19395-1795, Iran. \\ ${ }^{c}$ Research Institute for Fundamental Sciences, Tabriz 51664, Iran.
}

November 15, 2018

*E-mail:akhtarshenas@tabrizu.ac.ir

${ }^{\dagger}$ E-mail:jafarizadeh@tabrizu.ac.ir 


\begin{abstract}
We propose a simple geometrical approach for finding the robustness of entanglement for Bell decomposable states of $2 \otimes 2$ quantum systems. It is shown that the robustness of entanglement is equal to the concurrence. We also present an analytical expression for two separable states that wipe out all entanglement of these states. Finally the random robustness of these states is also obtained.
\end{abstract}

Keywords: Quantum entanglement, Bell decomposable states, Robustness of entanglement, Concurrence

PACs Index: 03.65.Ud 


\section{Introduction}

During the past decade an increasing study has been made on the entanglement, although it was discovered several decades ago by Einstein and SchrÖdinger [1, 2]. This is because of the central role that entanglement plays in the theory of quantum information [3, 4, 5. Entanglement as the most non classical features of quantum mechanics is usually arised from quantum correlations between separated subsystems which can not be created by local actions on each subsystem. By definition, a mixed state $\rho$ of a bipartite system is said to be separable (non entangled) if it can be written as a convex combination of pure product states

$$
\rho=\sum_{i} p_{i}\left|\phi_{i}^{A}\right\rangle\left\langle\phi_{i}^{A}|\otimes| \psi_{i}^{B}\right\rangle\left\langle\psi_{i}^{B}\right|
$$

where $\left|\phi_{i}^{A}\right\rangle$ and $\left|\psi_{i}^{B}\right\rangle$ are pure states of subsystems $A$ and $B$, respectively. Although, in the case of pure states of bipartite systems it is easy to check whether a given state is, or is not entangled, the question is yet an open problem in the case of mixed states.

There is also an increasing attention in quantifying entanglement, particularly for mixed states of a bipartite system, and a number of measures have been proposed [5, 6, 7, 8]. Among them the entanglement of formation has more importance, since it intends to quantify the resources needed to create a given entangled state.

Also a useful quantities which is introduced in [9] as a measure of entanglement is the robustness of entanglement. It corresponds to the minimal amount of mixing with separable states which washes out all entanglement. Analytical expression for pure states of binary systems have given in [9]. Authors in [10] gave a geometrical interpretation of robustness and pointed that two corresponding separable states needed to wipe out all entanglement are necessarily on the boundary of separable set. Unfortunately, above mentioned quantity as most proposed measures of entanglement 
involves extremization which are difficult to handle analytically.

In this paper we consider Bell decomposable (BD) states. We provide a simple geometrical approach and we give an analytic expression for robustness of entanglement and show that the corresponding separable states are on the boundary of separable states as pointed out in 10]. Our approach of determination of robustness of entanglement is geometrically intuitive. It is shown that for considered states the robustness is equal to the concurrence of states. Finally we obtain random robustness for BD states.

The paper is organized as follows. In section 2 we review BD states and we present a perspective of their geometry. The robustness of entanglement of these states is obtained in section 3 via a geometrical approach. Finally the random robustness is obtained in section 4 . The paper is ended with a brief conclusion.

\section{Bell decomposable states}

In this section we briefly review Bell decomposable (BD) states and some of their properties. A BD state is defined by:

$$
\rho=\sum_{i=1}^{4} p_{i}\left|\psi_{i}\right\rangle\left\langle\psi_{i}\right|, \quad 0 \leq p_{i} \leq 1, \quad \sum_{i=1}^{4} p_{i}=1
$$

where $\left|\psi_{i}\right\rangle$ is Bell state, given by:

$$
\begin{aligned}
& \left|\psi_{1}\right\rangle=\left|\phi^{+}\right\rangle=\frac{1}{\sqrt{2}}(|\uparrow \uparrow\rangle+|\downarrow \downarrow\rangle), \\
& \left|\psi_{2}\right\rangle=\left|\phi^{-}\right\rangle=\frac{1}{\sqrt{2}}(|\uparrow \uparrow\rangle-|\downarrow \downarrow\rangle), \\
& \left|\psi_{3}\right\rangle=\left|\psi^{+}\right\rangle=\frac{1}{\sqrt{2}}(|\uparrow \downarrow\rangle+|\downarrow \uparrow\rangle), \\
& \left|\psi_{4}\right\rangle=\left|\psi^{-}\right\rangle=\frac{1}{\sqrt{2}}(|\uparrow \downarrow\rangle-|\downarrow \uparrow\rangle) .
\end{aligned}
$$


In terms of Pauli's matrices, $\rho$ can be written as,

$$
\rho=\frac{1}{4}\left(I \otimes I+\sum_{i=1}^{3} t_{i} \sigma_{i} \otimes \sigma_{i}\right),
$$

where

$$
\begin{aligned}
& t_{1}=p_{1}-p_{2}+p_{3}-p_{4}, \\
& t_{2}=-p_{1}+p_{2}+p_{3}-p_{4}, \\
& t_{3}=p_{1}+p_{2}-p_{3}-p_{4} .
\end{aligned}
$$

From the positivity of $\rho$ we get

$$
\begin{aligned}
& 1+t_{1}-t_{2}+t_{3} \geq 0 \\
& 1-t_{1}+t_{2}+t_{3} \geq 0 \\
& 1+t_{1}+t_{2}-t_{3} \geq 0 \\
& 1-t_{1}-t_{2}-t_{3} \geq 0
\end{aligned}
$$

These equations form a tetrahedral with its vertices located at $(1,-1,1),(-1,1,1),(1,1,-1)$, $(-1,-1,-1)$ [13]. In fact these vertices denote the Bell states given in Eqs. (2-3) to (2-6), respectively.

According to the Peres and Horodecki's condition for separability [11, 12], a 2-qubit state is separable if and only if its partial transpose is positive. This implies that $\rho$ given in Eq. (2-7) is separable if and only if $t_{i}$ satisfy Eq. (2-9) and,

$$
\begin{aligned}
& 1+t_{1}+t_{2}+t_{3} \geq 0 \\
& 1-t_{1}-t_{2}+t_{3} \geq 0 \\
& 1+t_{1}-t_{2}-t_{3} \geq 0 \\
& 1-t_{1}+t_{2}-t_{3} \geq 0
\end{aligned}
$$


Inequalities (2-9) and (2-10) form an octahedral with its vertices located at $O_{1}^{ \pm}=( \pm 1,0,0)$, $O_{2}^{ \pm}=(0, \pm 1,0)$ and $O_{3}^{ \pm}=(0,0, \pm 1)$. So, tetrahedral of Eqs. (2-9) is divided into five regions. Central regions, defined by octahedral, are separable states. There are also four smaller equivalent tetrahedral corresponding to entangled states. Each tetrahedral takes one Bell state as one of its vertices. Three other vertices of each tetrahedral form a triangle which is its common face with octahedral (See Fig. 1).

\section{Robustness of entanglement}

According to [9] for a given entangled state $\rho$ and separable state $\rho_{s}$, a new density matrix $\rho(s)$ can be constructed as,

$$
\rho(s)=\frac{1}{s+1}\left(\rho+s \rho_{s}\right), \quad s \geq 0
$$

where it can be either entangled or separable. It was pointed that there always exits the minimal $s$ corresponding to $\rho_{s}$ such that $\rho(s)$ is separable. This minimal s is called the robustness of $\rho$ relative to $\rho_{s}$, denoted by $R\left(\rho \| \rho_{s}\right)$. The absolute robustness of $\rho$ is defined as the quantity,

$$
R(\rho \| S) \equiv \min _{\rho_{s} \in S} R\left(\rho \| \rho_{s}\right)
$$

Du et al. in [10] gave a geometrical interpretation of robustness and pointed that if $s$ in Eq. (3-11) is minimal among all separable states $\rho_{s}$, i.e. $s$ is the absolute robustness of $\rho$, then $\rho_{s}$ and $\rho(s)$ in Eq. (3-11) are necessarily on the boundary of the separable states.

Here in this section we obtain the absolute robustness for all Bell diagonal states, and we give an explicit form for the corresponding $\rho_{s}$ and $\rho(s)$ which are on the boundary of the separable states. 
Let us consider Fig. 2, we connect $t$, which denotes density matrix $\rho$, to the center of octahedral such that it cuts the plane $x_{1}+x_{2}+x_{3}+1=0$ (the boundary of separable octahedral) at $t^{\prime}$. Then we extend this segment, so that it cuts the other plane $x_{1}+x_{2}+x_{3}-1=0$ at $t^{\prime \prime}$.

These three points are along the same line but they posses different lengths. Also it is not difficult to see that they also lie on planes $x_{1}+x_{2}+x_{3}+\eta=0, x_{1}+x_{2}+x_{3}+1=0$ and $x_{1}+x_{2}+x_{3}-1$, respectively. Using the above argument, we arrive after some elementary algebra at the following results,

$$
\begin{array}{r}
t_{i}^{\prime}=\frac{t_{i}}{\eta}=\frac{-t_{i}}{t_{1}+t_{2}+t_{3}} \\
t_{i}^{\prime \prime}=\frac{-t_{i}}{\eta}=\frac{t_{i}}{t_{1}+t_{2}+t_{3}} .
\end{array}
$$

Now using the convexity of the set of density matrices, we can write $\rho_{s}^{\prime}$ as,

$$
\rho^{\prime}=\frac{1}{1+s}\left(\rho+s \rho^{\prime \prime}\right)
$$

where parameter $s$, called the robustness of $\rho$, can be written as

$$
s=\frac{\left|t t^{\prime}\right|}{\left|t^{\prime} t^{\prime \prime}\right|}=\frac{1+t_{1}+t_{2}+t_{3}}{2}=C,
$$

where $C$ is the concurrence of $\rho$ [8]. The numerical calculations indicate that thus obtained quantity is minimal with respect to all separable planes of the octahedral.

In the pioneering paper [9], robustness of entanglement of Werner states (as a particular kind of BD states), has been obtained from an entirely different approach. We see that thus obtained robustness of entanglement of Werner states via our proposed procedure is in agreement with the results of Reference 9 .

Finally, we would like to emphasis that our treatment is capable to give an explicit expression 
for the separable matrices $\rho_{s}^{\prime}$ and $\rho_{s}^{\prime \prime}$. Since, using Eqs. (3-13) and (2-7) we can write $\rho_{s}^{\prime}$ and $\rho_{s}^{\prime \prime}$ as

$$
\begin{gathered}
\rho_{s}^{\prime}=\frac{1}{4\left(t_{1}+t_{2}+t_{3}\right)}\left(\begin{array}{cccc}
t_{1}+t_{2} & 0 & 0 & -t_{1}+t_{2} \\
0 & t_{1}+t_{2}+2 t_{3} & -t_{1}-t_{2} & 0 \\
0 & -t_{1}-t_{2} & t_{1}+t_{2}+2 t_{3} & 0 \\
-t_{1}+t_{2} & 0 & 0 & t_{1}+t_{2}
\end{array}\right) \\
\rho_{s}^{\prime \prime}=\frac{1}{4\left(t_{1}+t_{2}+t_{3}\right)}\left(\begin{array}{cccc}
t_{1}+t_{2}+2 t_{3} & 0 & 0 & t_{1}-t_{2} \\
0 & t_{1}+t_{2} & t_{1}+t_{2} & 0 \\
0 & t_{1}+t_{2} & t_{1}+t_{2} & 0 \\
t_{1}-t_{2} & 0 & 0 & t_{1}+t_{2}+2 t_{3}
\end{array}\right)
\end{gathered}
$$

\section{Random Robustness}

Also Vidal And Tarrach [9] have defined another quantity so called random robustness, which is defined as robustness of $\rho$ relative to maximally random state $I / n$. For Bell decomposable states considered here we can evaluate it as follows. Using the convexity of the set of density matrices, we can write $\rho_{s}^{\prime}$ as,

$$
\rho^{\prime}=\frac{1}{1+s_{0}}\left(\rho+s_{0} \rho_{0}\right)
$$

where $\rho_{0}=\frac{I}{4}$ and

$$
s_{0}=\frac{\left|t t^{\prime}\right|}{\left|t^{\prime} O\right|}=-\left(1+t_{1}+t_{2}+t_{3}\right)=2 C,
$$

is random robustness of $\rho$. Note that for the states considered here, the separable matrix $\rho_{s}^{\prime}$ has the same form as given in Eq. (3-17) but with $\rho_{s}^{\prime \prime}=\frac{I}{4}$. 


\section{Conclusion}

We have obtained in this work the robustness of entanglement for Bell decomposable states. It is shown that the corresponding separable states which wipe out all entanglement of the states are on the boundary of separable states. The random robustness of these states is also obtained.

\section{References}

[1] A. Einstein, B. Podolsky And Rosen, Phys. Rev. 47, 777 (1935).

[2] E. SchrÖDInger, Naturwissenschaften. 23807 (1935).

[3] C. H. Bennett, and S. J. Wiesner, Phys. Rev. Lett. 69, 2881 (1992).

[4] C. H. Bennett, G. Brassard, C. Crépeau, R. Jozsa, A Peres and W. K. Wootters, Phys. Rev. Lett. 70, 1895 (1993).

[5] C. H. Bennett, D. P. Divincenzo, J. A. Smolin and W.K. Wootters, Phys. Rev. A 54, $3824(1996)$.

[6] V. Vedral, M. B. Pienio, M. A. Rippin and P. L. Knight Phys. Rev. Lett. 78, 2275 (1995).

[7] V. Vedral and M. B. Plenio, Phys. Rev. A 57, 1619 (1998).

[8] W. K. Wootters, Phys. Rev. Lett. 80 2245 (1998).

[9] G. Vidal and R. Tarrach, Phys. Rev. A 59, 141 (1999).

[10] J.F. Du, M.J. Shi, X.Y. Zhou and R.D. Han, Phys. Lett. A 267, 244 (2000). 
[11] A. Peres, Phys. Rev. Lett. 771413 (1996).

[12] M. Horodecki, P. Horodecki and R. Horodecki, Phys. Lett. A 2231 (1996).

[13] R. Horodecki and M. Horodecki Phys. Rev. A 541838 (1996). 


\section{Figure Captions}

Figure 1: All BD states are defined as points interior to tetrahedral. Vertices $P_{1}, P_{2}, P_{3}$ and $P_{4}$ denote projectors corresponding to Bell states given in Eqs. 2-3) to (2-6), respectively. Octahedral corresponds to separable states.

Figure 2: Entangled tetrahedral corresponding to singlet state. Point $t$ denotes a generic state $\rho$ and points $t^{\prime}$ and $t^{\prime \prime}$ are on the separable boundary planes defined by equations $x_{1}+x_{2}+x_{3}+1=0$ and $x_{1}+x_{2}+x_{3}-1=0$, respectively. 


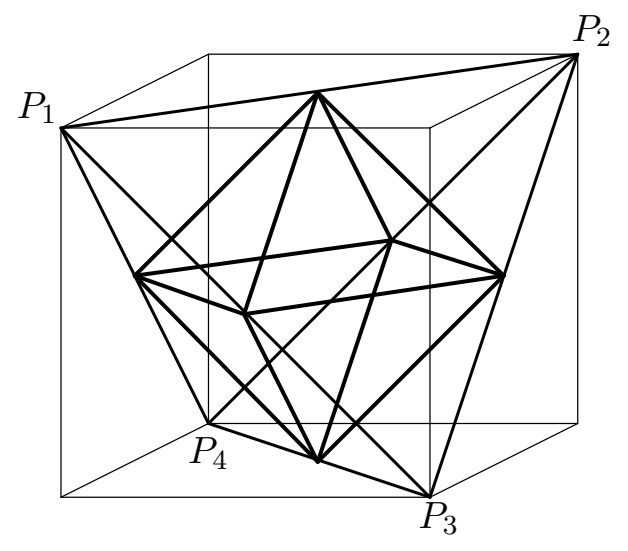

Figure 1: 


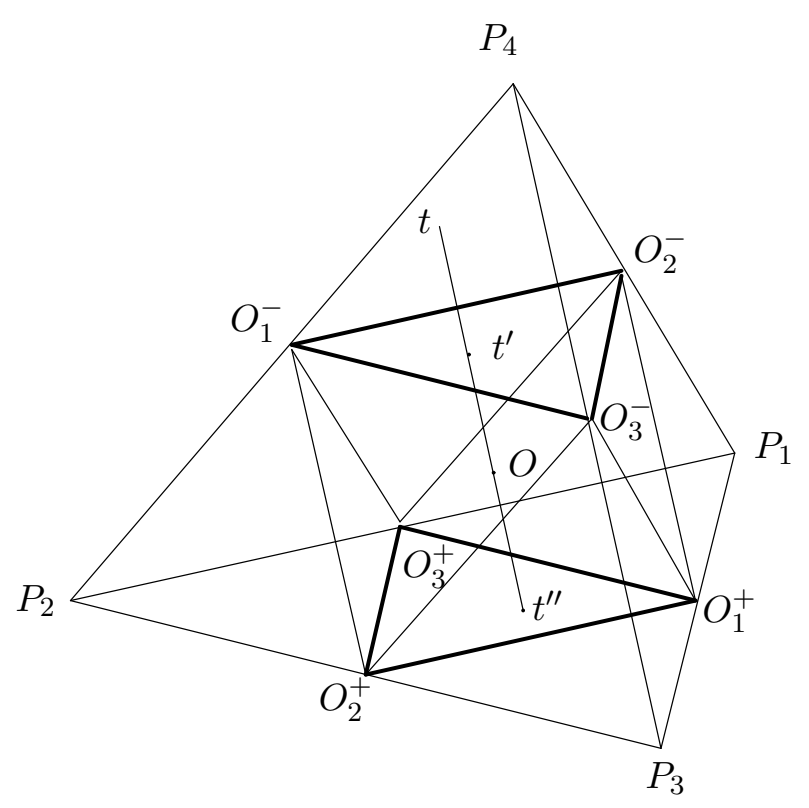

Figure 2: 